\title{
Volitional pursed lips breathing in patients with stable chronic obstructive pulmonary disease improves exercise capacity
}

Surya P. Bhatt

T. K. Luqman-Arafath

Anant Mohan

Tanujit Dey

William \& Mary

\section{Recommended Citation}

Bhatt, S. P., Luqman-Arafath, T. K., Gupta, A. K., Mohan, A., Stoltzfus, J. C., Dey, T., ... \& Guleria, R. (2013). Volitional pursed lips breathing in patients with stable chronic obstructive pulmonary disease improves exercise capacity. Chronic Respiratory Disease, 10(1), 5-10. 


\title{
Volitional pursed lips breathing in patients with stable chronic obstructive pulmonary disease improves exercise capacity
}

Chronic Respiratory Disease

10(I) 5-10

(C) The Author(s) 2012

Reprints and permission:

sagepub.co.uk/journalsPermissions.nav DOI: | 0.1 I77/|4799723 | 2464244

crd.sagepub.com

(S)AGE

\section{Surya P. Bhatt', T.K. Luqman-Arafath ${ }^{2}$, Arun K. Gupta ${ }^{3}$, Anant Mohan ${ }^{2}$, Jill C. Stoltzfus ${ }^{4}$, Tanujit Dey ${ }^{5}$, Sudip $\mathrm{Nanda}^{6}$ and Randeep Guleria ${ }^{2}$}

\begin{abstract}
Pursed lips breathing (PLB) is used by a proportion of patients with chronic obstructive pulmonary disease (COPD) to alleviate dyspnea. It is also commonly used in pulmonary rehabilitation. Data to support its use in patients who do not spontaneously adopt PLB are limited. We performed this study to assess the acute effects of PLB on exercise capacity in nonspontaneously PLB patients with stable COPD. We performed a randomized crossover study comparing 6-min walk test (6MWT) at baseline without PLB with 6WMT using volitional PLB. Spirometry, maximal inspiratory and expiratory mouth pressures, and diaphragmatic excursion during tidal and vital capacity breathing using B-mode ultrasonography were measured at baseline and after 10 min of PLB. A Visual Analog Scale (VAS) assessed subjective breathlessness at rest, after 6MWT and after 6MWT with PLB. $p \leq 0.0 \mathrm{I}$ was considered significant. Mean \pm SD age of patients was $53.1 \pm 7.4$ years. Forced expiratory volume in I second was I.I $\pm 0.4 \mathrm{~L} / \mathrm{min}(38.4 \pm 13.2 \%$ predicted). Compared with spontaneous breathing, all but one patient with PLB showed a significant increment in $6 \mathrm{MW}$ distance $(+34.9 \pm 26.4 \mathrm{~m} ; p=0.002)$. There was a significant reduction in respiratory rate post 6MWT with PLB compared with spontaneous breathing $(-4.4 \pm 2.8$ per minute; $p=0.003$ ). There was no difference in VAS scores. There was a significant correlation between improvement in 6MWT distance and increase in diaphragmatic excursion during forced breathing. The improvement was greater in patients who had poorer baseline exercise performance. PLB has an acute benefit on exercise capacity. Sustained PLB or short bursts of PLB may improve exercise capacity in stable COPD.
\end{abstract}

\section{Keywords}

COPD, pursed lips breathing, rehabilitation, 6-min walk test, exercise capacity

\section{Introduction}

Patients with chronic obstructive pulmonary disease (COPD) in respiratory distress frequently use pursed lips breathing (PLB) to alleviate dyspnea. Multiple mechanisms might be responsible for this perceived benefit, both at rest and on exertion. PLB at rest has been shown in various studies to improve gas exchange, ${ }^{1}$ increase efficiency of ventilation, ${ }^{2}$ and reduce respiratory rate (RR). ${ }^{3}$ PLB might reduce intrinsic positive end expiratory pressure (PEEP) by a way of generating positive pressures at the mouth and serving as a physiological extrinsic PEEP. By slowing expiration, it decreases the tendency of the airways to collapse by reducing the Bernoulli effect created by airflow. ${ }^{4}$ Dyspnea on exertion is associated
I University of lowa Hospital, Pulmonary and Critical Care Medicine, lowa City, IA, USA

2 Department of Internal Medicine, All India Institute of Medical Sciences, New Delhi, India

${ }^{3}$ Department of Radiodiagnosis, All India Institute of Medical Sciences, New Delhi, India

${ }^{4}$ Department of Biostatistics and Research, Temple University/St Luke's Hospital, Bethlehem, PA, USA

${ }^{5}$ Department of Mathematics, The College of William \& Mary, Williamsburg, VA, USA

${ }^{6}$ Division of Cardiovascular Medicine, Department of Internal Medicine, Lehigh Valley Hospital, Allentown, PA, USA

Corresponding author:

Surya P. Bhatt, University of lowa Hospital, Pulmonary and Critical Care Medicine, 200 Hawkins Drive, C33F GH, lowa City, IA 52242, USA.

Email: surya-bhatt@uiowa.edu 
with the extent and rate of respiratory muscle contraction. ${ }^{5}$ Exercise also leads to dynamic hyperinflation in patients with COPD. ${ }^{6}$ It is posited that PLB, by reducing the RR and therefore dynamic hyperinflation, brings the diaphragm to a vantage position. ${ }^{7}$ Based on these, PLB has been commonly used as a breathing technique in pulmonary rehabilitation. However, data to support its use in patients who do not spontaneously adopt PLB are conflicting. ${ }^{3,7-9}$

The purpose of this study is to determine the effects of volitional PLB on exercise capacity in patients with COPD who did not spontaneously adopt PLB. In view of conflicting reports of benefit in previous studies using varying techniques, we also assessed its effects on respiratory mechanics and dyspnea and sought to identify which patients would benefit from PLB.

\section{Methods}

We conducted an open-labeled randomized crossover study. A total of 14 patients with moderate-to-severe stable COPD, defined as no exacerbations in the preceding 4 weeks, were recruited for the study, from an outpatient pulmonary clinic at a single tertiary care referral center. Diagnosis of COPD was based on the characteristic findings on history and examination with typical radiographic abnormalities and confirmed by pulmonary function tests (PFTs). ${ }^{10}$ Exacerbation was defined as two of the following: worsening dyspnea, increased expectoration and increased purulence of sputum. ${ }^{10}$ Baseline demographic variables were obtained for all patients. Duration of disease, as determined by total duration of symptoms, was recorded. Active smokers were defined as having smoked within the past 6 months. Patients with comorbidities, such as diabetes mellitus, hypertension, congestive heart failure, tuberculosis, bronchiectasis, and intercurrent respiratory illness, were excluded. Those with physical limitations or comorbidities such as angina and peripheral vascular disease which precluded them from performing a 6-min walk test (6MWT) were also excluded. Measurements were conducted in three phases.

In the first phase, the patients were subjected to baseline spirometry. ${ }^{11}$ Maximal inspiratory (MIP) and expiratory pressures (MEP) measured at the mouth were recorded. ${ }^{10}$ The best of the three readings was documented. Patients were instructed on how to apply PLB according to standard guidelines. ${ }^{8,12}$ Patients were directed to inhale through the nose with the mouth closed and then exhale slowly over 4-6seconds through pursed lips held in a whistling position without cheek puffing or forceful expiration. ${ }^{12}$ PLB was applied for $10 \mathrm{~min}$ at rest. At the end of $10 \mathrm{~min}$, spirometry, MIP, and MEP measurements were repeated.

In the second phase, the patients performed a 6MWT. ${ }^{10}$ A subjective measure of dyspnea was recorded using a Visual Analog Scale (VAS), both at the start and immediately after the 6MWT. Baseline clinical variables, such as RR, blood pressure, and oxygen saturation $\left(\mathrm{SaO}_{2}\right)$, were noted. $\mathrm{RR}$ was also measured in the first minute after completion of the 6MWT. Patients who spontaneously used PLB during exercise or in the resting phase were to be excluded. None of the patients recruited used PLB spontaneously, and hence all patients were included in the study. The first $6 \mathrm{MWT}$ was also used as a practice test. The patients then performed two more 6MWT while applying PLB and without applying PLB. The sequence of 6MWT with and without PLB was interchanged at random so that seven patients with PLB performed the test first and seven without. This was to minimize the learning effect that could confound result interpretation. The randomization was done by having alternate patients perform their first test 6-min walk distance (6MWD) without PLB, and the rest perform their first test 6MWD with volitional PLB. VAS score was documented at the start and immediately after the 6MWT.

In the third phase, diaphragmatic movements were assessed using real-time B-mode ultrasound in the supine position using validated techniques. ${ }^{13}$ Diaphragmatic excursion during normal tidal and forced vital capacity (FVC) maneuver breathing was measured by a single-blinded qualified radiologist (AKG). A fixed skin position on the right lateral chest wall on the anterior axillary line was chosen to obtain a longitudinal plane of the right hemidiaphragm, including the maximal renal bipolar length. This allowed identification of the adjacent posterior aspect of the hemidiaphragm. A craniocaudal displacement line was marked with a cursor at the midpoint of the kidney, and excursion of the hemidiaphragm measured along this line with another cursor at the same depth from the transducer. Diaphragmatic excursion was measured both during the tidal breathing and during a vital capacity maneuver. ${ }^{13}$ For each maneuver, at least three satisfactory readings were taken. The better of the two values that agreed the most was selected for tidal breathing, and the best of the three efforts was chosen for forced breathing. All measurements were repeated after $10 \mathrm{~min}$ of PLB. All three phases were 
completed on the same day, with adequate rest in between phases, as indicated by patients returning to baseline VAS scores. An additional hour of rest was provided in between phases after return to baseline. Informed consent was obtained from all the patients. Ethical clearance was obtained from the Institutional Review Board.

\section{Statistical methods}

Descriptive data were recorded for all the patients. Due to small sample size, nonparametric tests were used for analyses. The mean values for tests performed before and after application of PLB (6WMD, VAS, respiratory mechanics, and ultrasound measures) were compared using the Wilcoxon signedrank test. Correlation was measured between the gradients in 6MWT distance and VAS, and the change in variables thought to significantly affect 6MWT distance and VAS scores [forced vital capacity (FVC), forced expiratory volume in one second $\left(\mathrm{FEV}_{1}\right)$, $\mathrm{FEV}_{1} / \mathrm{FVC}$, MIP, MEP, diaphragmatic excursion during tidal and deep breathing and respiratory rate] using nonparametric Spearman's correlation test. In view of the small sample size, a $p$ value of $\leq 0.01$ was considered significant for all analyses. All analyses were done using SPSS version 11.5 (SPSS Inc., Chicago, Illinois, USA).

\section{Results}

The anthropometric, demographic, and baseline PFT variables of the study population are given in Table 1 . A majority of patients were in Stages 3 and 4 according to the global initiative for chronic obstructive lung disease (GOLDCOPD) criteria. ${ }^{14}$ Most of the patients were active smokers or had a history of chronic smoking, as defined by at least 20 pack-years of smoking. All four nonsmokers had a history of significant exposure to biomass fuel.

Compared with the distance covered in 6MWT without application of PLB, there was a significant improvement in the 6MWD when PLB was used. Figure 1 shows the effects for each of the 14 patients. There was a significant reduction in RR after the 6MWT with PLB when compared with that without. A marginal decline was seen in the subjective assessment of dyspnea. This was however not statistically significant. Eight patients showed a decrease in VAS score, whereas 1 showed no change. Table 2 shows the effect of PLB on PFT parameters. When effects of delta change in respiratory test parameters with
Table I. Baseline demographic characteristics. ${ }^{a}$

\begin{tabular}{lc}
\hline Variable & $n=14$ \\
\hline Age (years) & $53.1 \pm 7.4$ (range 45-70) \\
Sex (\% males) & $10(7 I)$ \\
Median disease duration (years) & 3 (range I-8) \\
Smoker & 5 \\
Current & 5 \\
Ex & 4 \\
No & $19(0-72)$ \\
Pack-years & $48.9 \pm 10.8$ \\
FEV $/$ FVC & $1.09 \pm 0.4$ \\
FEV (L) & $38.4 \pm 13.2$ \\
FEV $\%$ & \\
GOLDCOPD stage & 0 \\
I & 4 \\
II & 5 \\
III & 5 \\
IV &
\end{tabular}

$\mathrm{FEV}_{1}$ : forced expiratory volume in Isecond; FVC: forced vital capacity; GOLDCOPD: global initiative for chronic obstructive lung disease to improve awareness and care of chronic obstructive pulmonary disease.

${ }^{\text {a }}$ Values are expressed as mean \pm SD or in absolute numbers.

application of PLB on the improvement in 6MWD were assessed, there was a good correlation with the change in forced diaphragmatic excursion with 6MWD ( $r=0.58, p=0.03$; see Figure 2$)$. We observed that patients with worse functional capacity showed a visibly larger increase in diaphragmatic excursion after PLB. When only patients with Stages 3 and 4 COPD were analyzed $(n=10)$, there was a greater correlation between change in diaphragmatic excursion on deep breathing and the improvement in 6MWD $(r=0.78$, $p=0.008)$. There was also a negative correlation between the improvement in 6MWD and the baseline $6 \mathrm{MWD}(r=-0.80, p=0.0007$; see Figure 3).

\section{Discussion}

We found that applying PLB in nonspontaneously PLB patients with COPD can increase exercise capacity. There was a marginal decrease in subjective sense of dyspnea. We also showed a decrease in the RR in the recovery period after exercise with PLB.

COPD is a progressive airway disease with few interventions that significantly improve quality of life. Despite widespread use of PLB in pulmonary rehabilitation, few studies have actually shown consistent benefit in imposing PLB in patients who do not already use it for relief. ${ }^{7,9,15}$ Our study is the first to almost uniformly demonstrate an improvement 




Figure I. Comparison of 6MWD before and after PLB. $X$ axis denotes individual patients. PLB: pursed lips breathing; 6MWD: 6-min walk distance.

Table 2. Lung function studies ${ }^{\mathrm{a}}$

\begin{tabular}{|c|c|c|c|}
\hline & Pre-PLB & Post-PLB & $p$ Value $^{a}$ \\
\hline $\mathrm{FEV}_{\mathrm{I}}(\mathrm{L})$ & $1.09 \pm 0.4$ & $1.17 \pm 0.45$ & 0.55 \\
\hline FEV $\% \%$ & $38.43 \pm 13.2$ & $41.29 \pm 14.64$ & 0.47 \\
\hline FVC (L) & $2.22 \pm 0.67$ & $2.21 \pm 0.70$ & 0.29 \\
\hline $\mathrm{FEV}_{1} / \mathrm{FVC}$ & $48.93 \pm 10.84$ & $52.29 \pm 10.07$ & 0.61 \\
\hline MMFR (L) & $0.59 \pm 0.26$ & $0.72 \pm 0.43$ & 0.61 \\
\hline MIP (mmHg) & $77.29 \pm 20.0$ & $74.64 \pm 17.84$ & 0.48 \\
\hline $\operatorname{MEP}(\mathrm{mmHg})$ & $74.0 \pm 22.96$ & $76.36 \pm 22.26$ & 0.25 \\
\hline Tidal excursion $(\mathrm{cm})$ & $2.01 \pm 0.71$ & $2.23 \pm 0.57$ & 0.12 \\
\hline Forced excursion $(\mathrm{cm})$ & $4.13 \pm 1.94$ & $4.63 \pm 1.43$ & 0.14 \\
\hline $6 M W D(m)^{c}$ & $410.11 \pm 89.85$ & $445.04 \pm 72.31$ & 0.002 \\
\hline VAS after 6MWT $(\mathrm{mm})$ & $30.86 \pm 0.68$ & $28.036 \pm 18.85$ & 0.38 \\
\hline RR after 6MWT (per minute) ${ }^{c}$ & $23.5 \pm 3.25$ & $19.29 \pm 4.90$ & 0.002 \\
\hline
\end{tabular}

$\mathrm{FEV}_{1}$ : forced expiratory volume in I second; FVC: forced vital capacity; MMFR: midmaximal flow rate; MIP: maximal inspiratory pressure; MEP: maximal expiratory pressure, 6MWD: 6-min walk distance; VAS: Visual Analog Scale; 6MWT: 6-min walk test; RR: respiratory rate; PLB: pursed lips breathing.

${ }^{\text {a }}$ All values are expressed as mean $\pm \mathrm{SD}$.

${ }^{\mathrm{b}} \mathrm{p}$ value calculated using Wilcoxon signed-rank test.

${ }^{c} p \leq 0.01$.

in functional exercise tolerance with PLB. Increase in functional exercise tolerance means a reduced work of breathing for the same level of effort effectively reducing the sense of dyspnea. This, however, probably comes at the cost of increased diaphragmatic activity leading to a sense of increase in the muscle activity, counterbalancing the subjective perception of reduced dyspnea. ${ }^{16}$ This could explain the relatively modest improvement in dyspnea scores in our and prior studies.

The effect on dyspnea has not been uniform across studies. This implies that there are responders and nonresponders within this class of nonspontaneous
PLB patients. Identification of responders will be valuable in exercise prescriptions. Bianchi et al. assessed the effect of PLB on subjective sense of dyspnea using the Borg score and sought to explain this by a change in operational lung volume, using optoelectronic plethysmography. ${ }^{7}$ They found that Borg score variability was significantly affected by a decrease in end expiratory volume of the chest wall. Spahija et al. demonstrated the variability in dyspnea perception with change in the end expiratory lung volume and the inspiratory muscle strength, using plethysmography and esophageal balloons. ${ }^{8}$ There was however a divergent response to PLB in their 


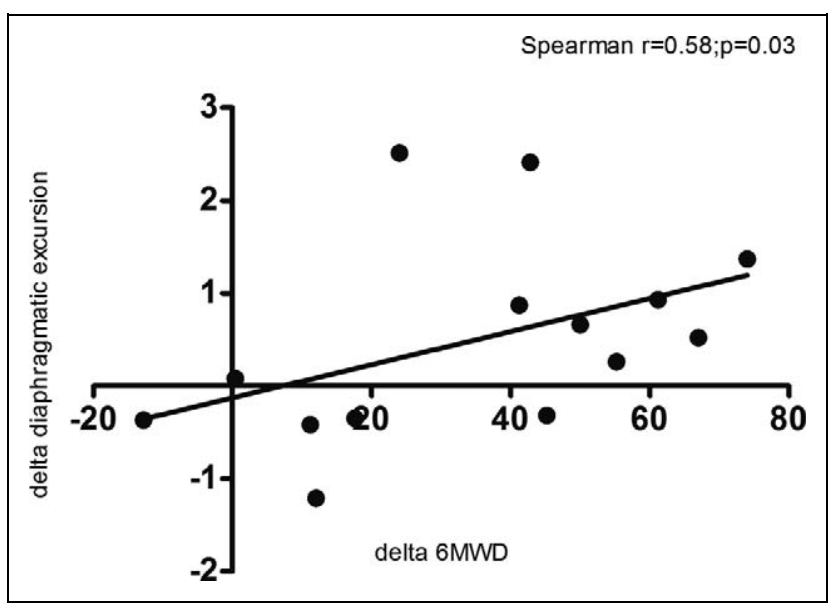

Figure 2. Correlation between change in 6MWD in meters with PLB and change in diaphragmatic excursion during vital capacity maneuver in centimeters. PLB: pursed lips breathing; 6MWD: 6-min walk distance.



Figure 3. Correlation between change in 6MWD and baseline 6MWD in meters. 6MWD: 6-min walk distance.

patient cohort. Garrod et al. could not show any change in dyspnea perception or in 6MWD. ${ }^{3}$ In contrast to these studies, we sought to identify responders to volitional application of PLB. After PLB, the delta change in forced diaphragmatic excursion correlated significantly with change in 6MWD. Deep breathing is more likely to be reflective of diaphragmatic function during exercise or respiratory distress. This might be a novel noninvasive way of identifying responders to PLB, although small numbers precluded us from defining cutoff values.

In our patients, there was a marginal reduction in subjective perception of dyspnea as indicated by the VAS. In fact, 8 of the 14 patients perceived a benefit and one showed no change. No single factor can possibly explain these changes. There was a significant decline in the RR during the recovery phase from the 6MWT at which time the VAS was marked. This is novel as most previous studies of PLB showed reduction in RR while applying PLB during recovery. ${ }^{1-3,7,8,17-19}$ Although this would occur by the nature of instructions for PLB, we showed that the RR is decreased in the period immediately after exertion with PLB. Hyperinflation is greater during periods of exertion because the increased frequency of breathing unfortunately results in proportionally reduced expiratory time leading to further air trapping and the onset of a vicious cycle. Slower controlled breathing during PLB reduces the rate and interrupts this cycle. This might translate into a lesser central neural drive, a lesser dissociation between actual ventilatory signals and the perceived effort (neuroventilatory dissociation), and a lesser perception of dyspnea. ${ }^{20}$ Grandevia has shown that PLB leads to a $20 \%$ increase in expired lung volume compared with a forced expiration, thereby reducing air trapping. ${ }^{21}$ The reduction in hyperinflation by reduction in the end expiratory lung volume results in an improved ability to increase the tidal volume for a given effort. ${ }^{6}$ The reduced RR also possibly allows more time for V/Q matching. ${ }^{1}$ The results of our study further refine the studies supporting the benefit of PLB in advanced COPD. ${ }^{1,3,9}$

Our study was limited by the small number of patients. We sought to alleviate this by applying a more stringent $p$ value $(\leq 0.01)$ for statistical significance. We did not directly measure the end expiratory lung volume and trans-diaphragmatic pressures generated to explain the changes. However, this is the first study to document diaphragmatic movement during PLB in real time by ultrasonography.

In summary, PLB increases functional exercise tolerance. This effect could be mediated by a reduction in RR and increased diaphragmatic movement.

\section{Authors' contribution}

SPB: conceived the study, acquired data, analyzed data, performed statistical analyses, and wrote manuscript; TKL: conceived the study, acquired data, and revised the manuscript for important intellectual content; AKG: performed ultrasound and revised the manuscript for important intellectual content; JCS and TD: performed statistical analyses and revised the manuscript for important intellectual content; $\mathrm{AM}$ and $\mathrm{SN}$ : revised the manuscript for important 
intellectual content; RG: conceived the study and revised the manuscript for important intellectual content.

\section{Funding}

This research received no specific grant from any funding agency in the public, commercial, or not-for-profit sectors.

\section{Conflicts of interest}

The authors declare no conflicts of interest.

\section{References}

1. Mueller RE, Petty TL and Filley GF. Ventilation and arterial blood gas changes induced by pursed lips breathing. J Appl Physiol 1970; 28: 784-789.

2. Thoman RL, Stoker GL and Ross JC. The efficacy of pursed-lips breathing in patients with chronic obstructive pulmonary disease. Am Rev Respir Dis 1966; 93: $100-106$.

3. Garrod R, Dallimore K, Cook J, et al. An evaluation of the acute impact of pursed lips breathing on walking distance in nonspontaneous pursed lips breathing chronic obstructive pulmonary disease patients. Chron Respir Dis 2005; 2: 67-72.

4. Schmidt RW, Wasserman K and Lillington GA. The effect of air flow and oral pressure on the mechanics of breathing in patients with asthma and emphysema. Am Rev Respir Dis 1964; 90: 564-571.

5. Leblanc P, Bowie DM, Summers E, et al. Breathlessness and exercise in patients with cardiorespiratory disease. Am Rev Respir Dis 1986; 133: 21-25.

6. O'Donnell DE. Hyperinflation, dyspnea, and exercise intolerance in chronic obstructive pulmonary disease. Proc Am Thorac Soc 2006; 3: 180-184.

7. Bianchi R, Gigliotti F, Romagnoli I, et al. Chest wall kinematics and breathlessness during pursed-lip breathing in patients with COPD. Chest 2004; 125: 459-465.

8. Spahija J, de Marchie M and Grassino A. Effects of imposed pursed-lips breathing on respiratory mechanics and dyspnea at rest and during exercise in COPD. Chest 2005; 128: 640-650.

9. Nield MA, Soo Hoo GW, Roper JM, et al. Efficacy of pursed-lips breathing: a breathing pattern retraining strategy for dyspnea reduction. J Cardiopulm Rehabil Prev 2007; 27: 237-244.
10. Standards for the diagnosis and care of patients with chronic obstructive pulmonary disease. American Thoracic Society. Am J Respir Crit Care Med 1995; 152: S77-S121.

11. Miller MR, Hankinson J, Brusasco V, et al. Standardisation of spirometry. Eur Respir J 2005; 26: 319-338.

12. Pulmonary rehabilitation-1999. American Thoracic Society. Am J Respir Crit Care Med 1999; 159: 1666-1682.

13. Houston JG, Angus RM, Cowan MD, et al. Ultrasound assessment of normal hemidiaphragmatic movement: relation to inspiratory volume. Thorax 1994; 49: 500-503.

14. Pauwels RA, Buist AS, Calverley PM, Jenkins CR and Hurd SS. Global strategy for the diagnosis, management, and prevention of chronic obstructive pulmonary disease. NHLBI/WHO Global Initiative for Chronic Obstructive Lung Disease (GOLD) Workshop summary. Am J Respir Crit Care Med 2001; 163:1256-76.

15. Martin $A D$ and Davenport PW. Extrinsic threshold PEEP reduces post-exercise dyspnea in COPD patients: a placebo-controlled, double-blind cross-over study. Cardiopulm Phys Ther J 2011; 22: 5-10.

16. Fitting JW, Chartrand DA, Bradley TD, et al. Effect of thoracoabdominal breathing patterns on inspiratory effort sensation. J Appl Physiol 1987; 62: 1665-1670.

17. Breslin EH. The pattern of respiratory muscle recruitment during pursed-lip breathing. Chest 1992; 101: 75-78.

18. Jones AY, Dean E and Chow CC. Comparison of the oxygen cost of breathing exercises and spontaneous breathing in patients with stable chronic obstructive pulmonary disease. Phys Ther 2003; 83: 424-431.

19. Tiep BL, Burns M, Kao D, et al. Pursed lips breathing training using ear oximetry. Chest 1986; 90: 218-221.

20. O'Donnell DE. Breathlessness in patients with chronic airflow limitation. Mechanisms and management. Chest 1994; 106: 904-912.

21. Grandevia B. The spirogram of gross expiratory tracheobronchial collapse in emphysema. $Q \mathrm{~J}$ Med 1963; 32: 23-31. 